\title{
Study Day of Tumor Findings
}

National Cancer Institute

\section{Source}

National Cancer Institute. Study Day of Tumor Findings. NCI Thesaurus. Code C119928.

The study day that a tumor findings assessment is performed. 